\title{
Self-accelerating two-step Steffensen-type methods with memory and their applications on the solution of nonlinear BVPs
}

\author{
Quan Zheng, Xiuhui Guo, Fengxi Huang \\ College of Sciences, North China University of Technology, Beijing 100144, China \\ zhengq@ncut.edu.cn (Q. Zheng)
}

\begin{abstract}
In this paper, seven self-accelerating iterative methods with memory are derived from an optimal two-step Steffensen-type method without memory for solving nonlinear equations, their orders of convergence are proved to be increased from 4 to $2+\sqrt{6},(5+\sqrt{17}) / 2,5$ and $(5+\sqrt{33}) / 2$, numerical examples are demonstrat-ed demonstrated to verify the theoretical results, and applications for solving systems of nonlinear equations and BVPs of nonlinear ODEs are illustrated.
\end{abstract}

Keywords-Nonlinear equation; Newton's method; Steffensen-type method; Derivative free; Super convergence

\section{Introduction}

Considering iterative methods to solve a nonlinear equation $f(x)=0$, the most famous method is Newton's method (NM, see $[1])$ :

$$
x_{n+1}=x_{n}-\frac{f\left(x_{n}\right)}{f^{\prime}\left(x_{n}\right)}, n=1,2, \ldots,
$$

where $x_{0}$ is an initial guess of the root. If the derivative $f^{\prime}\left(x_{n}\right)$ is

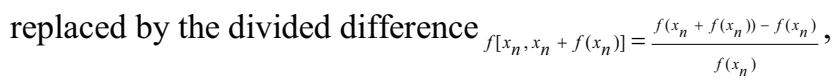

Newton's method becomes Steffensen's method (SM, see [1]). Steffensen's method is a tough competitor of Newton's method since it is derivative free. Because Kung and Traub conjectured in 1974 that a multipoint iteration based on m evaluations without memory has optimal order $2^{m-1}$ of convergence (see [2]), $\mathrm{NM}$ and SM are one-step methods of optimal order without memory. The efficiency index of them is $\sqrt{2}=1.4142$.

Further, a parametric Steffensen's method (PSM) was suggested in Section 8.4 in [3]:

$$
x_{n+1}=x_{n}-\frac{f\left(x_{n}\right)}{f\left[x_{n}, x_{n}+\beta_{n} f\left(x_{n}\right)\right]}, n=0,1,2, \ldots
$$

where $\beta \mathrm{n}$ are arbitrary parameters. PSM is convergent with the asymptotic convergence constant $\frac{\left(1+\beta_{n} f^{\prime}(a)\right) f^{\prime \prime}(a)}{2 f^{\prime}(a)}$. If $\beta_{n} \approx-\frac{1}{f^{\prime}(a)}$,

PSM can have smaller error. Therefore, by defining $\beta_{n}=-1 / f\left[x_{n-1}, x_{n-1}+\beta_{n-1} f\left(x_{n-1}\right)\right]$ at the previous iteration recursively as the iteration proceeds, a self-accelerating Steffensen's method (SASM) was proposed in Section 8.6 in [3]:

$$
\left\{\begin{array}{l}
x_{n+1}=x_{n}-\frac{f\left(x_{n}\right)}{f\left[x_{n}, x_{n}+\beta_{n} f\left(x_{n}\right)\right]}, \\
\beta_{n}=-\frac{1}{f\left[x_{n-1}, z_{n-1}\right]},
\end{array}\right.
$$

where $\bar{\beta}_{0}=-\operatorname{sign}\left(f^{\prime}\left(x_{0}\right)\right)$, or $-1 / f\left[x_{0}, x_{0}+f\left(x_{0}\right)\right]$, etc. SASM is a derivative-free one-step method with memory. It only uses two new evaluations of the function per step to achieve convergence of order $1+\sqrt{2} \approx 2.4142$, and has efficiency index $\sqrt{1+\sqrt{2}} \approx 1.5538$.

Some other optimal multipoint Steffensen-type methods without memory were derived in [4-7]. General optimal derivative-free iterative methods for solving nonlinear equations were introduced in [2] and [7]. Two-step selfaccelerating Steffensen-type methods were investigated in [810]. Steffensen-type methods and their applications in the solution of non-linear systems and nonlinear differential equations were discussed in the literature (see $[1,3,4,8,9]$ ).

This work suggests seven self-accelerating iterative methods with memory for solving nonlinear equations in section 2, proves their high orders of super convergence in section 3, demonstrates examples and applications in section 4, and makes conclusions in section 5 .

\section{The self-accelerating methods}

By using second-order Newtonian interpolation $N_{2}(x)$ after an iteration of SM to approximate $f(x)$ and find the root of $N_{2}(x)=0$ to approximate the exact root, we obtain a parametric Steffensen-type method (PSTM):

$$
\left\{\begin{array}{l}
y_{n}=x_{n}-\frac{f\left(x_{n}\right)}{f\left[x_{n}, z_{n}\right]}, \\
x_{n+1}=y_{n}-\frac{f\left(y_{n}\right)}{f\left[x_{n}, y_{n}\right]+f\left[y_{n}, z_{n}\right]-f\left[x_{n}, z_{n}\right]} .
\end{array}\right.
$$

where $z_{n}=x_{n}+\beta_{n} f\left(x_{n}\right)$. PSTM is an optimal two-step meth-od without memory (see [5, 7]).

Supported by Beijing Natural Science Foundation (No. 1122014). 
Theorem 2.1 (see [7]) Let $f: D \rightarrow R$ be a sufficiently differentiable function with a simple root $a \in D, D \subset R$ be an open set,

$x_{0}$ be close enough to $a$, then the method (4) is at least of fourth-order, and satisfies the error equation

$$
e_{n+1}=\left(1+\beta_{n} f^{\prime}(a)\right)^{2} c_{2}\left[c_{2}^{2}-c_{3}\right] e_{n}^{4}+O\left(e_{n}^{5}\right)
$$

where $c_{k}=\frac{f^{(k)}(a)}{k ! f^{\prime}(a)}, e_{n}=x_{n}-a, n=0,1,2, \ldots$

From PSTM and its error equation, in order to achieve super fourth-order convergence, the free parameters $\beta_{n}$ should tend to $-1 / f^{\prime}(a)$. Therefore, we propose six self-accelerating Steffensen-type methods (SASTMs) with memory as follows: compute (4) with

$$
\begin{array}{ll}
\text { (i) } \beta_{n} \equiv \beta_{n}^{1}=-\frac{1}{f\left[x_{n-1}, z_{n-1}\right]} & \text { (SASTM1) } \\
\text { (ii) } \beta_{n} \equiv \beta_{n}^{2}=-\frac{1}{f\left[x_{n-1}, y_{n-1}\right]} & \text { (SASTM2) } \\
\text { (iii) } \beta_{n} \equiv \beta_{n}^{3}=-\frac{1}{f\left[y_{n-1}, z_{n-1}\right]} & \text { (SASTM3) } \\
\text { (iv) } \beta_{n} \equiv \beta_{n}^{4}=-\frac{1}{f\left[x_{n-1}, x_{n}\right]} & \text { (SASTM4) } \\
\text { (v) } \beta_{n} \equiv \beta_{n}^{5}=-\frac{1}{f\left[z_{n-1}, x_{n}\right]} & \text { (SASTM5) } \\
\text { (vi) } \beta_{n} \equiv \beta_{n}^{6}=-\frac{1}{f\left[y_{n-1}, x_{n}\right]} & \text { (SASTM6) }
\end{array}
$$

By Newton's interpolation formula on points $x_{n}, y_{n-1}$ and $x_{n-1}$ as Follows:

$$
N(x)=f\left(x_{n}\right)+f\left[x_{n}, y_{n-1}\right]\left(x-x_{n}\right)+f\left[x_{n}, y_{n-1}, x_{n-1}\right]\left(x-x_{n}\right)\left(x-y_{n-1}\right)
$$

using $\beta_{n}=-1 / N^{\prime}\left(x_{n}\right)$ we propose the seventh self-accelerating Steffensen-type methods: compute (4) with

$$
\text { (vii) } \beta_{n} \equiv \beta_{n}^{7}=-\frac{1}{f\left[x_{n}, y_{n-1}\right]+f\left[x_{n}, x_{n-1}\right]-f\left[y_{n-1}, x_{n-1}\right]} \text {. }
$$

(SASTM7)

\section{The convergence}

Theorem 3.1. Let $f: D \rightarrow R$ be sufficiently differentiable near a simple root $a \in D, D \subset R$ be an open set, be close enough to $a$, then SASTM1, SASTM2 and SASTM4 achieve the convergence of order $2+\sqrt{6}$, SASTM3 and SASTM5 achieve the convergence of order $(5+\sqrt{17}) / 2$,SASTM6 achieves fifth- order convergence, and SASTM7 achieves the convergence of $\operatorname{order}(5+\sqrt{33}) / 2$.

Proof. Using Taylor formula and denoting $e_{n}^{z}=z_{n}-a$ and $e_{n}^{y}=y_{n}-a$, for SASTM1, we have

$$
e_{n}^{z}=\left(1+\beta_{n} f^{\prime}(a)\right) e_{n}+o\left(e_{n}\right)
$$

$$
f\left[x_{n}, z_{n}\right]=f^{\prime}(a)+\frac{f^{\prime \prime}(a)}{2}\left(2+\beta_{n} f^{\prime}(a)\right) e_{n}+o\left(e_{n}\right) .
$$

Hence,

$$
\beta_{n}^{1}=-\frac{1}{f^{\prime}(a)}\left[1-\left(2+\beta_{n-1}^{1} f^{\prime}(a)\right) c_{2} e_{n-1}\right]+o\left(e_{n-1}\right) .
$$

By substituting it into Eq. (5), we obtain

$$
e_{n+1}=\left(2+\beta_{n-1}^{1} f^{\prime}(a)\right)^{2} c_{2}^{3}\left[c_{2}^{2}-c_{3}\right] e_{n}^{4} e_{n-1}^{2}+o\left(e_{n-1}^{2} e_{n}^{4}\right) .
$$

For SASTM2, we have

$$
\begin{aligned}
& e_{n}^{y}=\left(1+\beta_{n} f^{\prime}(a)\right) c_{2} e_{n}^{2}+o\left(e_{n}^{2}\right), \\
& f\left[x_{n}, y_{n}\right]=f^{\prime}(a)+\frac{1}{2} f^{\prime \prime}(a) e_{n}+o\left(e_{n}\right), \\
& \beta_{n}^{2}=-\frac{1}{f^{\prime}(a)}\left[1-c_{2} e_{n-1}\right]+o\left(e_{n-1}\right) .
\end{aligned}
$$

By substituting $\beta_{n}^{2}$ into Eq. (5), we obtain

$$
e_{n+1}=c_{2}^{3}\left[c_{2}^{2}-c_{3}\right] e_{n}^{4} e_{n-1}^{2}+o\left(e_{n-1}^{2} e_{n}^{4}\right) .
$$

For SASTM4, we have

$$
f\left[x_{n-1}, x_{n}\right]=f^{\prime}(a)+\frac{1}{2} f^{\prime \prime}(a) e_{n-1}+o\left(e_{n-1}\right),
$$

and obtain the same error equation as the above. By solving $r^{2}=4 r+2$, the convergence of order $2+\sqrt{6} \approx 4.4495$ is proved for SASTM1, 2 and 4.

For SASTM3 and 5, if $z_{n}$ converges to $a$ with orderand $x_{n}$ Converges to $a$ with order $r$ as:

$$
e_{n}^{z}=C_{n} e_{n}^{p}+o\left(e_{n}^{p}\right) \text { and } e_{n+1}=D_{n} e_{n}^{r}+o\left(e_{n}^{r}\right),
$$

then

$$
\begin{aligned}
& e_{n}^{z}=C_{n} D_{n-1}^{p} e_{n-1}^{r p}+o\left(e_{n-1}^{r p}\right), \\
& e_{n+1}=D_{n} D_{n-1}^{r} e_{n-1}^{r^{2}}+o\left(e_{n-1}^{r^{2}}\right) .
\end{aligned}
$$

So, we have

$$
\begin{aligned}
& e_{n}^{y}=e_{n}-\frac{f\left[x_{n}, a\right] e_{n}}{f\left[x_{n}, z_{n}\right]}=\frac{f\left[x_{n}, z_{n}, a\right]}{f\left[x_{n}, z_{n}\right]} e_{n}^{z} e_{n}, \\
& e_{n+1}=e_{n}^{y}-\frac{f\left[y_{n}, a\right] e_{n}^{y}}{f\left[x_{n}, y_{n}\right]+f\left[x_{n}, y_{n}, z_{n}\right]\left(y_{n}-x_{n}\right)}
\end{aligned}
$$




$$
\begin{aligned}
& =e_{n}^{y} \frac{f\left[x_{n}, y_{n}, a\right]+f\left[x_{n}, y_{n}, z_{n}\right]\left(e_{n}^{y}-e_{n}\right)}{f\left[x_{n}, y_{n}\right]+f\left[x_{n}, y_{n}, z_{n}\right]\left(e_{n}^{y}-e_{n}\right)} \\
& =e_{n}^{y} \frac{f\left[x_{n}, y_{n}, z_{n}\right] e_{n}^{y}-f\left[x_{n}, y_{n}, z_{n}, a\right] e_{n}^{z} e_{n}}{f\left[x_{n}, y_{n}\right]+f\left[x_{n}, y_{n}, z_{n}\right]\left(e_{n}^{y}-e_{n}\right)} \\
& =\frac{f\left[x_{n}, z_{n}, a\right]}{f\left[x_{n}, z_{n}\right]}\left(e_{n}^{z} e_{n}\right)^{2} \\
& \quad \times \frac{f\left[x_{n}, y_{n}, z_{n}\right] \frac{f\left[x_{n}, z_{n}, a\right]}{f\left[x_{n}, z_{n}\right]}-f\left[x_{n}, y_{n}, z_{n}, a\right]}{f\left[x_{n}, y_{n}\right]+f\left[x_{n}, y_{n}, z_{n}\right]\left(e_{n}^{y}-e_{n}\right)} .
\end{aligned}
$$

For SASTM3,

$$
\begin{aligned}
e_{n}^{z} & =\frac{f\left[y_{n-1}, z_{n-1}\right]-f\left[x_{n}, a\right]}{f\left[y_{n-1}, z_{n-1}\right]} e_{n} \\
& =c_{2} e_{n-1}^{z} e_{n}+o\left(e_{n-1}^{z} e_{n}\right) \\
& =c_{2} C_{n-1} D_{n-1} e_{n-1}^{p+r}+o\left(e_{n-1}^{p+r}\right), \\
e_{n+1} & =c_{2}\left(c_{2} C_{n-1} D_{n-1}^{2} e_{n-1}^{p+2 r}\right)^{2}\left(c_{2}^{2}-c_{3}\right)+o\left(e_{n-1}^{2 p+4 r}\right) \\
& =c_{2}^{3}\left(c_{2}^{2}-c_{3}\right) C_{n-1}^{2} D_{n-1}^{4} e_{n-1}^{2 p+4 r}+o\left(e_{n-1}^{2 p+4 r}\right) .
\end{aligned}
$$

For SASTM5, we have the same results

$$
\begin{aligned}
& e_{n}^{z}=c_{2} C_{n-1} D_{n-1} e_{n-1}^{p+r}+o\left(e_{n-1}^{p+r}\right), \\
& e_{n+1}=c_{2}^{3}\left(c_{2}^{2}-c_{3}\right) C_{n-1}^{2} D_{n-1}^{4} e_{n-1}^{2 p+4 r}+o\left(e_{n-1}^{2 p+4 r}\right) .
\end{aligned}
$$

Comparing the exponents of $e_{n-1}$ in the expression ofand $e_{n+1}$ respectively, we have respectively, we have

$$
\left\{\begin{array}{c}
r p=p+r, \\
r^{2}=2 p+4 r .
\end{array}\right.
$$

From its non-trivial solution $p=(1+\sqrt{17}) / 2$ and $r=(5+\sqrt{17}) / 2$,

we prove that SASTM3 and 5 achieve the same convergence of $\operatorname{order}(5+\sqrt{17}) / 2 \approx 4.5616$.

For SASTM6 and 7, if

$$
e_{n}^{y}=C_{n} e_{n}^{p}+o\left(e_{n}^{p}\right) \text { and } e_{n+1}=D_{n} e_{n}^{r}+o\left(e_{n}^{r}\right)
$$

then

$$
\begin{aligned}
& e_{n}^{y}=C_{n} D_{n-1}^{p} e_{n-1}^{r p}+o\left(e_{n-1}^{r p}\right), \\
& e_{n+1}=D_{n} D_{n-1}^{r} e_{n-1}^{r^{2}}+o\left(e_{n-1}^{r^{2}}\right) .
\end{aligned}
$$

So, for SASTM6,

$$
\begin{aligned}
& e_{n}^{z}=c_{2} C_{n-1} D_{n-1} e_{n-1}^{p+r}+o\left(e_{n-1}^{p+r}\right), \\
& e_{n}^{y}=c_{2}^{2} C_{n-1} D_{n-1}^{2} e_{n-1}^{p+2 r}+o\left(e_{n-1}^{p+2 r}\right), \\
& e_{n+1}=c_{2}^{3}\left(c_{2}^{2}-c_{3}\right) C_{n-1}^{2} D_{n-1}^{4} e_{n-1}^{2 p+4 r}+o\left(e_{n-1}^{2 p+4 r}\right),
\end{aligned}
$$

and establish

$$
\left\{\begin{array}{c}
r p=p+2 r \\
r^{2}=2 p+4 r
\end{array}\right.
$$

From its non-trivial solution $p=5 / 2$ and $r=5$, we prove that SASTM6 achieves fifth-order convergence.

For SASTM7, we have

$$
\begin{aligned}
e_{n}^{z} & =\frac{f\left[x_{n}, y_{n-1}, a\right] e_{n-1}^{y}+f\left[x_{n}, y_{n-1}, x_{n-1}\right]\left(e_{n}-e_{n-1}^{y}\right)}{f\left[x_{n}, y_{n-1}\right]+f\left[x_{n}, x_{n-1}\right]-f\left[y_{n-1}, x_{n-1}\right]} e_{n} \\
& =\frac{f\left[x_{n}, y_{n-1}, x_{n-1}\right] e_{n}-f\left[x_{n}, y_{n-1}, x_{n-1}, a\right] e_{n-1}^{y} e_{n-1}}{f\left[x_{n}, y_{n-1}\right]+f\left[x_{n}, x_{n-1}\right]-f\left[y_{n-1}, x_{n-1}\right]} e_{n} \\
& =-c_{3} C_{n-1} D_{n-1} e_{n-1}^{p+r+1}+o\left(e_{n-1}^{p+r+1}\right), \\
e_{n}^{y} & =-c_{2} c_{3} C_{n-1} D_{n-1}^{2} e_{n-1}^{p+2 r+1}+o\left(e_{n-1}^{p+2 r+1}\right), \\
e_{n+1} & =c_{2} c_{3}^{2}\left(c_{2}^{2}-c_{3}\right) C_{n-1}^{2} D_{n-1}^{4} e_{n-1}^{2 p+4+2}+o\left(e_{n-1}^{2 p+4 r+2}\right),
\end{aligned}
$$

and establish

$$
\left\{\begin{array}{c}
r p=p+2 r+1, \\
r^{2}=2 p+4 r+2 .
\end{array}\right.
$$

From its non-trivial solution $p=(5+\sqrt{33}) / 4$ and $r=(5+\sqrt{33}) / 2 \approx 5.3723$, we prove that SASTM7 achieves the convergence of order $(5+\sqrt{33}) / 2$.

Each of SASTMs is a two-step derivative-free method with memory and only uses three new evaluations of the function per step to achieve super fourth-order convergence. SASTM1, 2 and 4 have the same efficiency index $\sqrt[3]{2+\sqrt{6}} \approx 1.6448$. SASTM3 and 5 have the same efficiency index $\sqrt[3]{(5+\sqrt{17}) / 2} \approx 1.6585$. SASTM 6 and 7 have the efficiency indices $\sqrt[3]{5} \approx 1.7100$ and $\sqrt[3]{(5+\sqrt{33}) / 2} \approx 1.7514$, respectively. Whereas, two self-accelerating methods proposed by Petković-Ilić-Džunić in[10] each uses three new evaluations of the function per iteration to achieve the super fourth-order convergence of order $2+\sqrt{6}$ and its efficiency index is only $\sqrt[3]{2+\sqrt{6}} \approx 1.6448$.

\section{Numerical examples}

In the examples,NM,SM,PSM,SASM, PSTM and SASTMs are compared with each other. The computational order of convergence is defined as:

$$
C O C=\frac{\log \left(\left|e_{n}\right| /\left|e_{n-1}\right|\right)}{\log \left(\left|e_{n-1}\right| /\left|e_{n-2}\right|\right)} .
$$

where $\beta_{\mathrm{o}}^{i}=1, i=1,2, \ldots, 7$.

Example 1. Numerical results in Table I agree with theoretical results in the theorems.

TABLE I. $f(x)=x^{2}-e^{-x}+3 x+1, a=0, x_{0}=0.2$

\begin{tabular}{|l|l|cccc|}
\hline Method & $\mathrm{n}$ & 1 & 2 & 3 & 4 \\
\hline NM & $\left|e_{n}\right|$ & $.533 \mathrm{e}-2$ & $.356 \mathrm{e}-5$ & $.158 \mathrm{e}-11$ & $.312 \mathrm{e}-24$ \\
& Coc & & 2.01691 & 2.00030 & 2.00000 \\
& & & & $.165 \mathrm{e}-6$ & $.170 \mathrm{e}-13$ \\
& $\left|e_{n}\right|$ & $.282 \mathrm{e}-1$ & $.513 \mathrm{e}-3$ & .16 & \\
& Coc & & 2.04367 & 2.00830 & 2.00009 \\
\hline
\end{tabular}




\begin{tabular}{|c|c|c|c|c|c|}
\hline $\begin{array}{l}\text { PSM } \\
\left(\beta_{n} \equiv-1\right)\end{array}$ & $\begin{array}{l}\left|e_{n}\right| \\
C O C\end{array}$ & $.134 \mathrm{e}-1$ & $\begin{array}{r}.677 \mathrm{e}-4 \\
1.95757\end{array}$ & $\begin{array}{c}.172 \mathrm{e}-8 \\
2.00059\end{array}$ & $\begin{array}{l}.111 \mathrm{e}-17 \\
2.00000\end{array}$ \\
\hline SASM & $\begin{array}{l}\left|e_{n}\right| \\
C O C\end{array}$ & $.282 \mathrm{e}-1$ & $\begin{array}{r}.160 \mathrm{e}-4 \\
3.81335\end{array}$ & $\begin{array}{l}.131 \mathrm{e}-12 \\
2.49109\end{array}$ & $\begin{array}{l}.433 \mathrm{e}-32 \\
2.40945\end{array}$ \\
\hline $\begin{array}{l}\text { PSTM } \\
\left(\beta_{n} \equiv 1\right)\end{array}$ & $\begin{array}{l}\left|e_{n}\right| \\
\operatorname{Coc}\end{array}$ & $.468 \mathrm{e}-4$ & $\begin{array}{l}.389 \mathrm{e}-18 \\
3.87748\end{array}$ & $\begin{array}{c}.186 \mathrm{e}-74 \\
4.00000\end{array}$ & $\begin{array}{r}.977 \mathrm{e}-300 \\
4.00000\end{array}$ \\
\hline $\begin{array}{l}\text { PSTM } \\
\left(\beta_{n} \equiv-1\right)\end{array}$ & $\begin{array}{l}\left|e_{n}\right| \\
C O C\end{array}$ & $.595 \mathrm{e}-4$ & $\begin{array}{l}.366 \mathrm{e}-18 \\
4.02926\end{array}$ & $\begin{array}{l}.525 \mathrm{e}-75 \\
4.00000\end{array}$ & $\begin{array}{r}.222 \mathrm{e}-302 \\
4.00000\end{array}$ \\
\hline SASTM1 & $\begin{array}{l}\left|e_{n}\right| \\
\operatorname{COC}\end{array}$ & $.468 \mathrm{e}-4$ & $\begin{array}{l}.414 \mathrm{e}-21 \\
4.69620\end{array}$ & $\begin{array}{c}.441 \mathrm{e}-98 \\
4.51372\end{array}$ & $\begin{array}{r}.331 \mathrm{e}-440 \\
4.44480\end{array}$ \\
\hline SASTM2 & $\begin{array}{l}\left|e_{n}\right| \\
C O C\end{array}$ & $.468 \mathrm{e}-4$ & $\begin{array}{l}.135 \mathrm{e}-22 \\
5.10548\end{array}$ & $\begin{array}{l}.371 \mathrm{e}-104 \\
4.39945\end{array}$ & $\begin{array}{r}.175 \mathrm{e}-467 \\
4.45460\end{array}$ \\
\hline SASTM3 & $\begin{array}{l}\left|e_{n}\right| \\
C O C\end{array}$ & $.468 \mathrm{e}-4$ & $\begin{array}{l}.317 \mathrm{e}-21 \\
4.72820\end{array}$ & $\begin{array}{l}.228 \mathrm{e}-100 \\
4.60962\end{array}$ & $\begin{array}{r}.956 \mathrm{e}-462 \\
4.56612\end{array}$ \\
\hline SASTM4 & $\begin{array}{l}\left|e_{n}\right| \\
\operatorname{COC}\end{array}$ & $.468 \mathrm{e}-4$ & $\begin{array}{r}.104 \mathrm{e}-22 \\
5.13642\end{array}$ & $\begin{array}{r}.132 \mathrm{e}-104 \\
4.39102\end{array}$ & $\begin{array}{r}.166 \mathrm{e}-469 \\
4.45547\end{array}$ \\
\hline SASTM5 & $\begin{array}{l}\left|e_{n}\right| \\
\operatorname{COC}\end{array}$ & $.468 \mathrm{e}-4$ & $\begin{array}{r}.302 \mathrm{e}-21 \\
4.73383\end{array}$ & $\begin{array}{r}.180 \mathrm{e}-100 \\
4.60889\end{array}$ & $\begin{array}{r}.324 \mathrm{e}-462 \\
4.56606\end{array}$ \\
\hline SASTM6 & $\begin{array}{l}\left|e_{n}\right| \\
C O C\end{array}$ & $.468 \mathrm{e}-4$ & $\begin{array}{r}.195 \mathrm{e}-24 \\
5.61230\end{array}$ & $\begin{array}{r}.691 \mathrm{e}-127 \\
5.02717\end{array}$ & $\begin{array}{r}.384 \mathrm{e}-639 \\
5.00000\end{array}$ \\
\hline SASTM7 & $\begin{array}{l}\left|e_{n}\right| \\
C O C\end{array}$ & $.468 \mathrm{e}-4$ & $\begin{array}{r}.810 \mathrm{e}-27 \\
6.26819\end{array}$ & $\begin{array}{r}.207 \mathrm{e}-148 \\
5.34210\end{array}$ & $\begin{array}{r}.679 \mathrm{e}-802 \\
5.37438\end{array}$ \\
\hline
\end{tabular}

Example 2. Consider to solve a boundary-value problem of ODEs as the following:

$$
\left\{\begin{array}{l}
y^{\prime \prime}=\sqrt{1+y^{\prime 2}}, \\
y(0)=1, y(1)=\frac{1}{2}\left(e+e^{-1}\right) .
\end{array}\right.
$$

Let $N=4$ and use the methods to solve the nonlinear system $F(\vec{s})=0$ with $\vec{s}_{0}=0$ by the multiple shooting method (see $[4,9]$ ). The numerical results are showed in Table II.

TABLE II.

FOR THE MULTIPLE SHOOTING METHOD

\begin{tabular}{|c|c|c|c|c|c|}
\hline Method & $\mathrm{n}$ & 1 & 2 & 3 & 4 \\
\hline \multirow[t]{3}{*}{ SM } & \multirow{3}{*}{$\begin{array}{l}\left\|\boldsymbol{F}\left(\overrightarrow{\boldsymbol{s}}_{n}\right)\right\| \\
\left\|y-y_{n}\right\| \\
\left\|y^{\prime}-y_{n}^{\prime}\right\|\end{array}$} & $.560 \mathrm{e}-1$ & $.149 \mathrm{e}-3$ & $.994 \mathrm{e}-10$ & $.203 \mathrm{e}-21$ \\
\hline & & $.283 \mathrm{e}-1$ & $.584 \mathrm{e}-4$ & $.326 e-5$ & $.326 e-5$ \\
\hline & & $.585 \mathrm{e}-1$ & $.143 \mathrm{e}-3$ & $.459 \mathrm{e}-5$ & $.458 \mathrm{e}-5$ \\
\hline \multirow[t]{3}{*}{ PSTM } & \multirow{3}{*}{$\begin{array}{l}\left\|F\left(\overrightarrow{\boldsymbol{s}}_{n}\right)\right\| \\
\left\|y-y_{n}\right\| \\
\left\|y^{\prime}-y_{n}^{\prime}\right\|\end{array}$} & $.271 \mathrm{e}-3$ & $.124 \mathrm{e}-17$ & $.219 \mathrm{e}-76$ & $.336 e-311$ \\
\hline & & $.142 \mathrm{e}-3$ & $.326 e-5$ & $.326 e-5$ & $.326 \mathrm{e}-5$ \\
\hline & & $.346 \mathrm{e}-3$ & $.459 \mathrm{e}-5$ & $.459 \mathrm{e}-5$ & $.458 \mathrm{e}-5$ \\
\hline \multirow[t]{3}{*}{ SASTM1 } & \multirow{3}{*}{$\begin{array}{l}\left\|F\left(\overrightarrow{\boldsymbol{s}}_{n}\right)\right\| \\
\left\|y-y_{n}\right\| \\
\left\|y^{\prime}-y_{n}^{\prime}\right\|\end{array}$} & $.271 \mathrm{e}-3$ & $.154 \mathrm{e}-4$ & $.280 \mathrm{e}-9$ & $.301 e-15$ \\
\hline & & $.142 \mathrm{e}-3$ & $.230 \mathrm{e}-5$ & $.326 e-5$ & $.326 e-5$ \\
\hline & & $.346 \mathrm{e}-3$ & $.129 \mathrm{e}-4$ & $.458 \mathrm{e}-5$ & $.458 \mathrm{e}-5$ \\
\hline \multirow[t]{2}{*}{ SASTM2 } & \multirow{2}{*}{$\begin{array}{l}\left\|F\left(\vec{s}_{n}\right)\right\| \\
\left\|y-y_{n}\right\| \\
\left\|y^{\prime}-y_{n}^{\prime}\right\|\end{array}$} & $.271 \mathrm{e}-3$ & $.135 \mathrm{e}-21$ & $.289 \mathrm{e}-99$ & $.118 \mathrm{e}-446$ \\
\hline & & $.142 \mathrm{e}-3$ & $.326 \mathrm{e}-5$ & $.326 \mathrm{e}-5$ & $.326 e-5$ \\
\hline
\end{tabular}

\begin{tabular}{|c|c|c|c|c|c|}
\hline & & $.346 \mathrm{e}-3$ & $.459 \mathrm{e}-5$ & $.459 \mathrm{e}-5$ & $.458 \mathrm{e}-5$ \\
\hline \multirow[t]{3}{*}{ SASTM3 } & \multirow{3}{*}{$\begin{array}{l}\left\|F\left(\overrightarrow{\boldsymbol{s}}_{n}\right)\right\| \\
\left\|y-y_{n}\right\| \\
\left\|y^{\prime}-y_{n}^{\prime}\right\|\end{array}$} & $.271 \mathrm{e}-3$ & $.764 \mathrm{e}-20$ & $.236 \mathrm{e}-95$ & $.121 \mathrm{e}-439$ \\
\hline & & $.142 \mathrm{e}-3$ & $.326 \mathrm{e}-5$ & $.326 \mathrm{e}-5$ & $.326 e-5$ \\
\hline & & $.346 \mathrm{e}-3$ & $.459 \mathrm{e}-5$ & $.459 \mathrm{e}-5$ & $.458 \mathrm{e}-5$ \\
\hline \multirow[t]{3}{*}{ SASTM4 } & \multirow{3}{*}{$\begin{array}{l}\left\|F\left(\overrightarrow{\boldsymbol{S}}_{n}\right)\right\| \\
\left\|y-y_{n}\right\| \\
\left\|y^{\prime}-y_{n}^{\prime}\right\|\end{array}$} & $.271 \mathrm{e}-3$ & $.446 \mathrm{e}-22$ & \multicolumn{2}{|c|}{$.131 \mathrm{e}-102.711 \mathrm{e}-462$} \\
\hline & & $.142 \mathrm{e}-3$ & $.326 \mathrm{e}-5$ & $.326 \mathrm{e}-5$ & $.326 \mathrm{e}-5$ \\
\hline & & $.346 \mathrm{e}-3$ & $.459 \mathrm{e}-5$ & $.459 \mathrm{e}-5$ & $.458 \mathrm{e}-5$ \\
\hline \multirow[t]{3}{*}{ SASTM5 } & \multirow{3}{*}{$\begin{array}{l}\left\|F\left(\vec{s}_{n}\right)\right\| \\
\left\|y-y_{n}\right\| \\
\left\|y^{\prime}-y_{n}^{\prime}\right\|\end{array}$} & $.271 \mathrm{e}-3$ & $.996 \mathrm{e}-20$ & $.897 \mathrm{e}-95$ & $.564 \mathrm{e}-437$ \\
\hline & & $.142 \mathrm{e}-3$ & $.326 \mathrm{e}-5$ & $.326 \mathrm{e}-5$ & $.326 \mathrm{e}-5$ \\
\hline & & $.346 \mathrm{e}-3$ & $.459 \mathrm{e}-5$ & $.459 \mathrm{e}-5$ & $.458 \mathrm{e}-5$ \\
\hline \multirow[t]{3}{*}{ SASTM6 } & \multirow{3}{*}{$\begin{array}{l}\left\|F\left(\overrightarrow{\boldsymbol{s}}_{n}\right)\right\| \\
\left\|y-y_{n}\right\| \\
\left\|y^{\prime}-y_{n}^{\prime}\right\|\end{array}$} & $.271 \mathrm{e}-3$ & $.135 \mathrm{e}-21$ & $.289 \mathrm{e}-99$ & $.118 \mathrm{e}-446$ \\
\hline & & $.142 \mathrm{e}-3$ & $.326 e-5$ & $.326 e-5$ & $.326 \mathrm{e}-5$ \\
\hline & & $.346 \mathrm{e}-3$ & $.459 \mathrm{e}-5$ & $.459 \mathrm{e}-5$ & $.458 \mathrm{e}-5$ \\
\hline \multirow[t]{3}{*}{ SASTM7 } & \multirow{3}{*}{$\begin{array}{l}\left\|F\left(\overrightarrow{\boldsymbol{s}}_{n}\right)\right\| \\
\left\|y-y_{n}\right\| \\
\left\|y^{\prime}-y_{n}^{\prime}\right\|\end{array}$} & $.271 \mathrm{e}-3$ & $.446 \mathrm{e}-22$ & $.131 \mathrm{e}-102$ & $.711 \mathrm{e}-462$ \\
\hline & & $.142 \mathrm{e}-3$ & $.326 \mathrm{e}-5$ & $.326 \mathrm{e}-5$ & $.326 \mathrm{e}-5$ \\
\hline & & $.346 \mathrm{e}-3$ & $.458 \mathrm{e}-5$ & $.458 \mathrm{e}-5$ & $.458 \mathrm{e}-5$ \\
\hline
\end{tabular}

\section{Conclusions}

In this work, we derive seven self-accelerating Steffensentype methods with memory for solving nonlinear equations and establish the comparative advantage of high efficiency theoretically and numerically. The suggested self-accelerating methods without derivative are convenient to be applied in the multiple shooting method for solving boundary-value problems of nonlinear ODEs, where derivatives are difficult to be obtained.

\section{REFERENCES}

[1] J.M. Ortega, W.G. Rheinboldt, Iterative Solution of Nonlinear Equations in Several Variables, Academic Press, New York, 1970.

[2] H.T. Kung, J.F. Traub, Optimal order of one-point and multipoint iteration, J. Assoc. Comput. Math. 21 (1974) 634-651.

[3] J.F. Traub, Iterative Methods for the Solution of Equations, Prentice-Hall, Englewood Cliffs, New Jersey, 1964.

[4] V. Alarcón, S. Amat, S. Busquier, D. J. López, A Stef-fensen's type method in Banach spaces with applications on boundaryvalue problems, J. Comput. Appl. Math. 216 (2008) 243-250.

[5] W. Bi, H. Ren, Q. Wu, A class of two-step Steffensen type methods with fourth-order convergence, Appl. Math. Comput. 209 (2009) 206-210.

[6] F. Soleymani, S.K. Vanani, Optimal Steffensen-type meth-ods with eighth order of convergence, Comput. Math. Appl. 62 (2011) 4619-4626.

[7] Q. Zheng, J. Li, F. Huang, An optimal Steffensen-type family for solving nonlinear equations, Appl. Math. Comput. 217 (2011) 9592-9597.

[8] Q. Zheng, J. Wang, P. Zhao, L. Zhang, A Steffensen-like method and its higher-order variants, Appl. Math. Comput. 214 (2009) 10-16.

[9] Q. Zheng, P. Zhao, L. Zhang, W. Ma, Variants of Steffensensecant method and applications, Appl. Math. Comput. 216 (2010) 3486-3496.

[10] M.S. Petković, S. Ilić, J. Džunić, Derivative free two-point methods with and without memory for solving nonlinear equations, Appl. Math. Comput. 217 (2010) 1887-1895. 